\title{
Innovative design based on intelligent mobile folding studio
}

\author{
Yang Qingmei ${ }^{1}$, Wang Lihui ${ }^{2}$ andZhang Yansong ${ }^{1 *}$ \\ 1,2,* Dalian Polytechnic University School of Fashion and Design, Liaoning Dalian, 116034, China
}

\begin{abstract}
This article introduces the interactive and operable working principle, basic composition and overall performance of intelligent mobile folding studios, and briefly reviews the development overview of mobile folding studios at home and abroad, and makes prospects and innovative designs for its future development requirements and market requirements. Designed an intelligent mobile folding studio suitable for the public and multi-regions, which can be used to meet the needs of office workers, workers and amateurs such as modern urban white-collar workers, and has also broken the limitations of long-term fixed regionalization. Comprehensive use of key technologies such as manufacturing and manipulation control, programming, intelligence, and 3D Max modeling software for 3D structural design, and based on this analysis of the application prospects and development trends of mobile folding studios.
\end{abstract}

\section{Advances in mobile folding studios}

Due to different economic systems, social environments and production conditions, different forms and scales of photo studios may be formed. The early studio was just a "big shed" with only ceilings and scaffolding, missing on all sides. China's "studio" name came from this. The original studio was only installed with wooden cubes at the temporary need of the ceiling string under the roof frame for the purpose of hanging the light board and fixing the scenery, which is very unsafe. Later, protective railings were gradually installed in or under the roof trusses for safety, but there were still inconveniences when working. Later it was developed into a grille-type ceiling, making the entire ceiling a workbench where various tasks can be performed everywhere, thereby making the work of scenery and lighting more flexible, effective, rapid and safe. The top of the grid can be made of wood, which has a light structure and low cost, but has poor fire resistance. It can also be made of steel or reinforced concrete, which is strong, fire-resistant, and has a large load bearing, but its own weight is too large to fix the scenery with nails. The use of metal connectors is both laborious and inflexible.

\section{Characteristics of intelligent mobile folding studio}

The intelligent mobile mobile folding studio uses various information input and output carriers as the hardware system, and uses a variety of carriers to comprehensively install and manufacture a controllable folding space scene as a shooting platform. It can realize the interactive mode of information control and has a strong comprehensive nature.

The biggest difference between intelligent and mobile folding studios and other studios lies in their movable spatial creativity, which is a three-dimensional entity occupying a certain space, can be integrated into the concept of time, and become an image creation and art display in the thinking spacetime. From the perspective of creative tools, the intelligent mobile folding studio is more complicated than ordinary traditional studios, including a variety of sensors, computer software and hardware, and decorative materials. Among them, programming is an important part of the intelligent mobile mobile folding studio. Partly, it is a key feature that is different from other traditional studio forms, in order to solve the problems of inconvenience for mobile carrying, unfavorable adjustment of light sources, poor structural strength and compression resistance, and poor safety.

\subsection{Composition of Intelligent Mobile Folding Studio}

The intelligent mobile folding studio adopts the idea of block design. According to the role of each part in the traditional studio shooting process, it can be divided into the following functional modules: 1. Base plate; 2. Card slot; 3. Back plate; 4. Movable buckle; 5. Light box; 6. Top plate; 7. Side plate; 8. Roller; 9. Background cloth; 10. Adjusting chain; 11. Lock handwheel; 12. Light plate; 13. Adjusting lever; 14. Super hydrophobic transparent coating; 15. Flame retardant layer; 16. Flame retardant ball; 17. Fire blocking material; 18. Compression layer; 19. Carbon steel reinforcement layer; 20. Reinforcing ribs; 21. Single-chip computer; 22. Bluetooth module; 23. Communication module; 24. Electric wire; 25. Prompt light, etc (see Figure 1-5).

\footnotetext{
*Corresponding author's e-mail: 1805742763@qq.com
} 


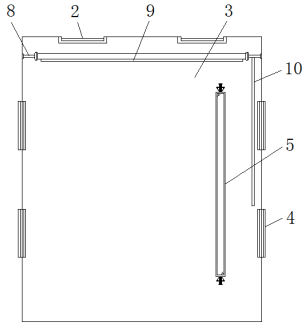

Figure 1.Backplane face-up structure.

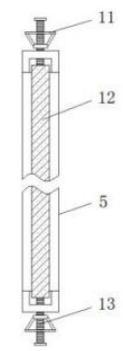

Figure 2. Light box section structure.

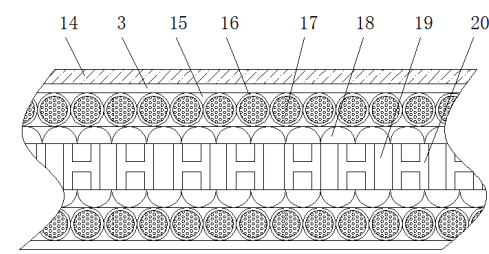

Figure 3. Back side wall cross-section structure.

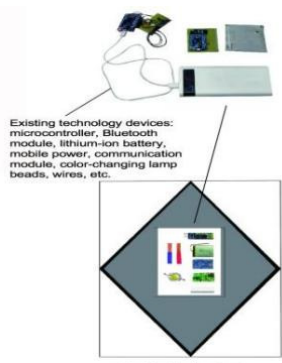

Figure 4. Interna structure.

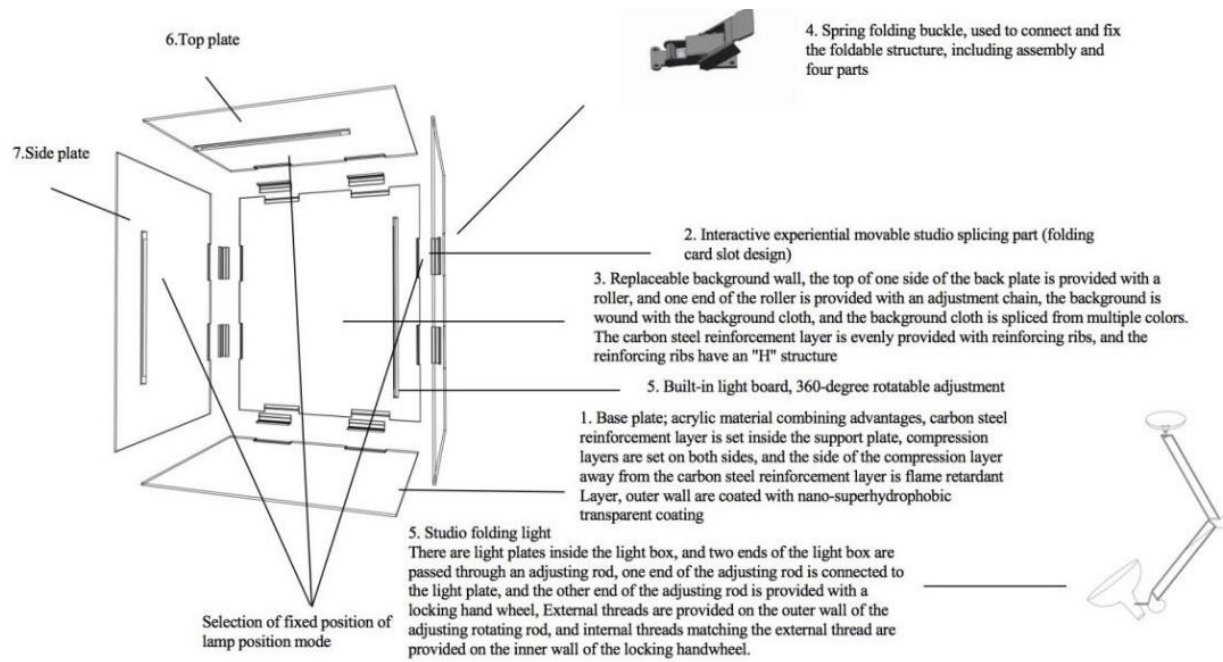

Figure 5. Three-dimensional structure of an intelligent mobile folding studio.

the old pattern of the traditional expression mode in the past, so that the traditional art form is gradually transformed by the promotion of new technology (see

\subsection{Intelligent mobile folding}

The rapid development of new media art and digital technology has gradually made the interactive form one of the popular art forms [1], and has also catalyzed the new technological change of intelligent mobile folding studios with interactive nature. With the improvement and improvement of technical level and means, the intelligent mobile folding studio not only provides the interaction between art works and participants, but also satisfies the sensory needs of modern people in terms of sight, hearing and touch. The distance has broken through Figure 6) [2]. The design of the intelligent mobile folding studio combined with the intelligence meets the daily shooting needs of the general public. The design of the folding space will be based on calculation and measurement, and the placement of the lights will be professionally adjusted. Make photos appear professional level, not limited to a single shooting mode. To create a new and unique spatial experience, to meet the audience's ever-changing aesthetic pursuit and the need for timeliness and convenience.

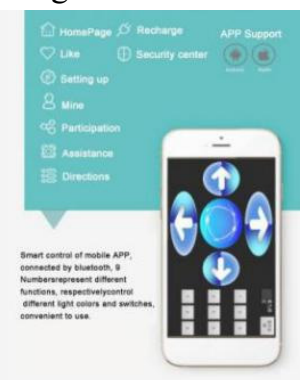

Figure 6. APP smart Operation.

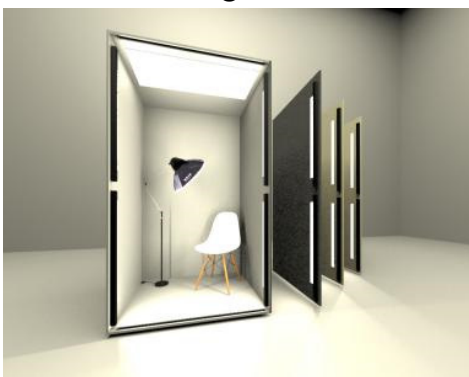

Figure 7. Model of the exterior structure of the studio.

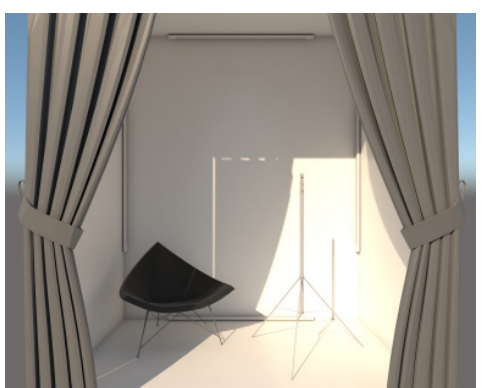

Figure 8. Model of theinterior structure of the studio. 


\subsection{Extending application scope}

The application of intelligent mobile folding studio technology is to innovate and improve the traditional studio model, and to build a new design experience and creation platform based on the current state of social development. Compared with traditional studios, the intelligent mobile folding studio has a lot of advanced features and advantages. Its exhibition format changes the original fixed old model, allowing viewers to appreciate and experience from different times and dimensions at any time, Take photos or evaluate, to further increase audience participation and enthusiasm. In this way, participants can better understand the designer's intention and the true value of the product. This participation method is also more in line with the current trend of social development.

\section{The key technology of intelligent mobile folding studio}

\subsection{Optimized Design}

The intelligent mobile folding studio is equipped with a back plate, a bottom plate, a top plate and a side plate of acrylic material, all of which are coated with a nano-superhydrophobic transparent coating on the outer wall. Cards are evenly arranged at the connection of the back plate, the bottom plate, the top plate and the side plate Slot, and the back plate, the bottom plate, the top plate and the side plates are evenly hinged by a movable buckle, so that the device can be folded and spliced, and can be adjusted according to the size of the indoor space. In addition, a top of one side of the back plate is provided. The roller of the adjustment chain is wound with a background cloth on the roller, which is convenient for the background to be assembled according to actual needs. The background cloth is stitched by a variety of colors. It can be freely selected according to shooting needs to meet a variety of shooting needs.

The intelligent mobile folding studio has a light box fixed on the back plate, the bottom plate, the top plate and the side plates. The light box is provided with a light plate inside. Both ends of the light box pass through an adjusting rod, and one end of the adjusting rod is connected with The light board is connected. The other end of the adjusting lever is provided with a locking hand wheel. The outer wall of the adjusting lever is provided with external threads. The inner wall of the locking hand wheel is provided with an internal thread matching the external thread. The lever can drive the light board to rotate $360^{\circ}$, and then screw the handwheel to fix the limit position, which is convenient for freely adjusting the light source illumination according to the shooting situation, and it is flexible to use.

\subsection{Compression, flame retardant and other safety technologies}

The intelligent mobile folding studio is provided with a carbon steel reinforcement layer inside the back plate, the bottom plate, the top plate and the side wall of the side plate, and the inside of the carbon steel reinforcement layer is evenly provided with " $\mathrm{H}$ " -shaped reinforcing ribs, which is convenient for improving the back plate. The overall strength of the bottom plate, the top plate, and the side plates. At the same time, the two sides of the carbon steel reinforcement layer are uniformly provided with a semi-arc-shaped compression layer, which is convenient for improving the compression performance of the back plate, the bottom plate, the top plate, and the side plates. It is not easy to be damaged at the same time. In addition, the intelligent mobile movable folding studio is provided with a flame retardant layer inside the back plate, the bottom plate, the top plate and the side plate side walls. The flame retardant balls are evenly arranged in the flame retardant layer. The inside of the ball is evenly filled with fire blocking material. The material of the fire blocking material is polycarbonate, which can effectively prevent the fire from spreading when it is exposed to fire and improve safety. At the same time, it is applied to the back, bottom, top, and side plates. The outside is coated with a nano-superhydrophobic transparent coating. Water will form spherical water droplets on the surface of the material. The water droplets will roll off under the effect of gravity. The rolling water droplets will remove some dust and sludge particles. Take away and achieve self-cleaning effect.

\subsection{Intelligent programming control technology}

The intelligent mobile folding studio adds the programming technology of intelligent APP software. It is a professional lighting mode that is easier to operate, making the control technology simpler and more efficient. Efficient work and autonomous decision-making. Intelligently control creation It has a good development space and prospects. The intelligent mobile folding studio is provided with a single-chip microcomputer, and the light position and warning lights are electrically connected to the single-chip microcomputer. The single-chip microcomputer is connected to the mobile terminal through the Bluetooth module. Switching and controlling the light color of the warning lamp have the comprehensive advantages of strong functionality, high portability, high cost performance, and the like.

\subsection{Fault diagnosis}

Failure of the lighting system in the programming project is inevitable, but there will be some signs before the failure occurs. Using intelligent technology can have a comprehensive and accurate judgment. Because the status of the transformer in the power equipment is very important, the monitoring of its operating status also seems to pay special attention. It often has irregular testing and maintenance, but this does not completely avoid the occurrence of faults and timely To diagnose faults, in order to minimize the losses caused by faults, the best choice is intelligent technology. This will not 
only find the cause of the fault as soon as possible, but also minimize the loss caused by it [3].

\section{Advantages and development prospects of intelligent mobile folding studios}

As a fast, efficient and convenient interactive and innovative design, the intelligent mobile folding studio conforms to the concepts of innovation, science and the times, allowing the experiencers to fully understand the new positioning of this multifunctional carrier, which is more perfect. It shows the modern and fashionable development of the city. The purpose is to deliver a functional service method that can be placed in densely populated places such as office buildings and community streets, which solves space and technical problems, saves valuable time, and greatly improves work efficiency. The concise shape design, eye-catching color matching, and clear information function are just the embodiment of modern city service level (see Figure 7-8) [4].

\section{Conclusion}

Intelligent mobile folding studio, as a new art form that can be created in a multi-dimensional and omnidirectional manner in time and space, shows the flexibility of time and space applications. The linear thinking originally restricted by the traditional studio has transformed into a new art form that appears interactively, breaking the traditional old pattern. Art has developed to this day. In the context of cross-border integration, artists can transform any available technology into the carrier and medium of creation, and break the boundaries of new artistic creation again and again. These selected new carriers are not only in art It has outstanding performance in expression, and integrates new impetus for artistic creation in the concept, while satisfying the experience and happiness of participating audiences.

\section{Fund Projects}

Scientific research project of education department of liaoning province (J2019036); 2019 University Students Innovation and Entrepreneurship Training Project (201910152172).

\section{References}

1. Wang, M.H. (2018) Research on interactive installation art noumenon. J. Western leather, 21:108.

2. $\mathrm{Xu}, \mathrm{G} .(2000)$ What is installation art. J. Art observation, 11:69-73.

3. Wang, H. (2016) Application and development of intelligent technology in automation control of electrical engineering in China. J. Natural science, $14: 181$.

4. Du, C.W. (2019) Design and application of interactive installation art in urban commercial space. D. Wuxi: Jiangnan University. 